\title{
Limits on the Detection of Transients Imposed by Scattering
}

\author{
Jean-Pierre Macquart* ${ }^{* \dagger}$ \\ NRAO/Caltech \\ E-mail: jpmeastro.caltech.edu
}

Three scattering mechanisms that limit the observable properties low-frequency emission from transient sources are discussed. Both Induced Compton and Induced Raman scattering potentially hinder the escape of the bright radiation envisaged to be generated from the compact extragalactic transients and that many hope to detect with future widefield radio telescopes. In cases where the radiation is observed, they constrain the nature of the mechanism responsible for the emission. Scattering in the Intergalactic Medium is a potentially powerful tool for probing the structure of all the ionized baryons in the IGM (in which most of the baryonic mass of the universe resides at $z \lesssim$ 7), but is also likely to strongly hinder the observability of short-timescale events at frequencies below $\sim 300 \mathrm{MHz}$. We apply this scattering physics to the transient recently reported by Lorimer et al. and discuss with the duration of the event is likely dominated by scattering in the IGM and not the local ISM. If this is the case, it implies that turbulence exists in the IGM down to scales less than $\sim 7 \times 10^{7} \mathrm{~m}$, and a simple argument suggests that the magnetic field in the IGM scattering region is $\gtrsim 4\left(T_{e} / 10^{3} \mathrm{~K}\right)^{1 / 2} \mathrm{nG}$.

Bursts, Pulses and Flickering: wide-field monitoring of the dynamic radio sky

12-15 June 2007

Kerastari, Tripolis, Greece

\footnotetext{
${ }^{*}$ Speaker.

$\dagger$ Jansky Fellow
} 


\section{Introduction}

Considerable attention has been devoted lately to the sorts of new radio transients one might be able to detect with the next generation of widefield radio arrays, such as LOFAR, the MWA, the LWA, ASKAP and, eventually, the SKA ${ }^{1}$. Such telescopes will open a swath of parameter space in the sensitivity and short-timescale domain that has hitherto been inaccessible. However, the expectation that searches of such large volumes of parameter space will automatically reveal new, exotic objects should be tempered by the possibility that new physics comes into play in this regime which precludes the escape of such emission. Nature may well generate the types of emission we aspire to detect, but it is another thing entirely for the radiation to be able to propagate to the observer.

This concern is particularly relevant to the extraordinarily bright, short timescale low-frequency radio emission proposed to emanate during the prompt phase of gamma-ray bursts (Usov \& Katz 2000; Sagiv \& Waxman 2002; Moortgat \& Kuijpers 2005). The exceptionally high $\left(\ggg 10^{20} \mathrm{~K}\right)$ brightness temperatures associated with their emission renders the radiation particularly susceptible to a number of nonlinear effects in which the plasma through which the emission must propagate back-reacts onto the radiation itself and scatters it. The two nonlinear plasma effects most likely to limit the properties of bright emission are induced Compton and induced Raman scattering. The substantial rôle played by these two mechanisms in limiting the observability of prompt GRB radio emission was discussed in Macquart (2007).

However, both induced Compton and induced Raman scattering are effective under a much wider range of circumstances than those merely relevant to GRBs. To illustrate that they are applicable to a wide variety of transient radio sources, I will discuss them here in the context of the specific SHort Extragalactic Radio Pulse (hereafter SHERP) recently reported by Lorimer et al. (2007). This $5 \mathrm{~ms}$ pulse was detected with a flux density of $30 \pm 10 \mathrm{Jy}$ at $1.4 \mathrm{GHz}$ with the Parkes Multibeam receiver and a dispersion measure (DM) of $375 \mathrm{~cm}^{-3} \mathrm{pc}$. Its DM is at least a factor of several larger than the expected contribution from the Milky Way and the Small Magellanic Cloud, near which the object was detected. The poor localisation of the source precluded association of the object with any specific background galaxy, but it was located sufficiently far from the SMC that it was unlikely to be associated with it. Lorimer et al. argue that the DM implies that the source is extragalactic and, based on a simple theoretical model of the Intergalactic Medium (Ioka 2003), is located at $\sim 500 \mathrm{Mpc}$. However, this distance is at best an educated guess given our complete ignorance of the distribution of ionized baryons in the Intergalactic Medium (IGM).

The $5 \mathrm{~ms}$ SHERP duration also bears implications for the effectiveness of temporal smearing due to multipath propagation through the inhomogeneous IGM. Multipath propagation can severely limit the detectability of transients at low $(<300 \mathrm{MHz})$ frequencies by smearing out the energy of a sharp pulse into an event that is so long that the pulse power falls below the threshold of detectability. Lorimer et al. argue that the remarkably short duration of the SHERP is most likely not its intrinsic duration but is instead due to temporal smearing caused by multipath propagation. The apparent pulse width scales as $v^{-4.8 \pm 0.4}$ across the $\sim 300 \mathrm{MHz}$ observing bandwidth, consistent with the expectation of temporal smearing.

\footnotetext{
${ }^{1}$ These acronyms correspond, respectively, to: LOw FRequency ARray, Murchison Widefield Array, LongWavelength Array, Australian SKA Pathfinder, and the Square Kilometre Array.
} 
Our discussion of the limiting effects of these three scattering processes is organised as follows. In the following section we apply the physics of induced Compton scattering to deduce some properties of the SHERP, and in section 3 we briefly discuss induced Raman scattering. In section 4 we investigate the implications of the observed $5 \mathrm{~ms}$ scattering duration in relation to prospects of detecting similar transients at lower frequencies. The conclusions are summarised in section 5 .

\section{Induced Compton scattering}

\subsection{The origin of the effect}

Induced Compton scattering is effective at scattering the radiation of extremely bright objects even if the Thomson optical depth to the source is small. The effect is associated with the stimulated scattering of photons to lower energy levels. A simple physical argument for the origin of the effect, given by Wilson \& Rees (1978), is as follows. Consider two photon energy states $a$ and $b$. The rate of change in the population of level $a$ due to scatterings from $a$ to $b$ is given by

$$
\frac{d n_{a-}}{d t} \propto-n_{a}(\omega)-n_{a}(\omega) n_{b}(\omega+\Delta \omega),
$$

where the first term is due to spontaneous scatterings, and the second component is due to induced emission, and $\Delta \omega=\hbar \omega^{2}(1-\cos \theta) / m_{e} c^{2}$ is the small frequency shift associated with the scattering and $\theta$ is the angle between the directions associated with $a$ and $b$. The change in the population of $a$ caused by scatterings from $b$ back to $a$ is

$$
\frac{d n_{a+}}{d t} \propto n_{a}(\omega)+n_{a}(\omega) n_{b}(\omega-\Delta \omega) .
$$

Thus the total population of level $a$ would not change were it not for the small frequency shift on scattering, which prevents the induced terms from cancelling exactly. Taylor-expanding in $\Delta \omega$, the depopulation rate of level $a$ is

$$
\frac{d n_{a}}{d t} \propto n_{b}-n_{a}+2 \Delta \omega n_{a} \frac{\partial n_{b}}{\partial \omega},
$$

from which it may be shown that induced scatterings dominate over spontaneous scatterings if the brightness temperature of the radiation satisfies $T_{B}>m_{e} c^{2} / k \Omega^{2}$, where $\Omega$ is the solid angle subtended by the radiation beam. Induced Compton scattering strongly scatters any radiation whose brightness exceeds ${ }^{2} T_{B} \approx m_{e} c^{2} /\left(k \tau_{T} \Omega^{2}\right)$. Now, in the special case of a relativistic source, the angular size of the source is always $\Gamma^{-1}$ and, after correcting all quantities for relativistic beaming, the induced Compton scattering imposes the following constraint to a source whose emission is isotropic in the rest frame but is relativistically beamed (Begleman, Ergun \& Rees 2006)

$$
\begin{aligned}
1 & >\frac{\tau_{T} k T_{B}}{m_{e} c^{2} \Gamma^{2}}, \\
1 & >3.44 \times 10^{20} \Gamma^{-2}\left(\frac{\mathrm{DM}}{100 \mathrm{pccm}^{-3}}\right)\left(\frac{T_{B}}{10^{34} \mathrm{~K}}\right),
\end{aligned}
$$

where we express the electron column (i.e. Thomson optical depth) in the conventional pulsar astronomers' units of dispersion measure and have normalised to the nominal brightness temperature deduced for the SHERP.

\footnotetext{
${ }^{2}$ Note that the exponent involving $\Delta \Omega$ in eq.(1) of Macquart 2007 should be -2 rather than 2.
} 


\subsection{Specific remarks regarding the SHERP}

Under certain circumstances induced Compton scattering poses a strong limitation on extremely bright emission even if the Thomson optical depth is small. If the emission from the SHERP were generated isotropically in its rest frame, eq. (2.5) would require the radiation to be extremely highly beamed, with a Lorentz factor, $\Gamma>4 \times 10^{10}$. This, in turn, would require the apparent beamsize, $\sim \Gamma^{-1}$, to be so small as to imply an enormous event rate. For every one object that one observes, there are up to another $4 \pi \Gamma$ objects that would potentially remain undetected. This number is reduced, however, if the emission we see comes from only part of a large concentrically expanding front that subtends a large solid angle.

There are several extra caveats that apply in particular to the SHERP. The first is that, if its emission is intrinsically collimated and emanates from a small region (as occurs for pulsar emission), then the angular size $\Omega$ of the source as viewed from the scattering region can be very much smaller than that dictated by relativistic beaming alone. This is almost certainly the case here if the emission mechanism generates radiation that is already partially collimated. Such an argument obviously applies to any sort of coherent maser process, such as curvature maser emission (Luo \& Melrose 1995), to name but one example. Indeed, we remark that in the extreme limit, a perfectly collimated beam is not subject to induced Compton scattering.

Pulsars, whose brightness temperatures easily exceed $10^{30} \mathrm{~K}$, are readily observed and thus appear to evade induced Compton scattering. It is instructive to consider the two main reasons for this. The first is that the emission region is small (and partially collimated), so $\Omega$ is extremely small, thus partially alleviating the limitation on the brightness temperature. However, this by itself is not enough for induced Compton scattering to imply a trivial limitation on the scattering medium surrounding the pulsar. Wilson \& Rees (1978) showed that the absence of induced Compton scattering associated with high brightness temperature emission observed from the Crab pulsar implies that its wind's Lorentz factor must exceed $10^{4}$. This high Lorentz factor requirement arises in order reduce the cross section to scattering near the source of the emission (where $\Omega$ is large). Indeed, Wilson \& Rees (1978) showed that the absence of induced Compton scattering associated with high brightness temperature emission observed from the Crab pulsar implies that its wind's Lorentz factor must exceed $10^{4}$. Similar limits likely apply to any material immediately surrounding the SHERP.

\section{Induced Raman scattering}

Induced Raman scattering is an effect wherein a beam of sufficiently high energy density propagating through a dense plasma drives in an instability which generates Langmuir waves in the plasma. The resulting Langmuir turbulence then back-reacts onto the beam and scatters it (e.g. Levinson \& Blandford 1995). This effect is most likely to be important in the region immediate to the source, where high plasma densities may occur and where the energy density of the incident emission is extremely large.

A necessary condition for effective induced Raman scattering is that the plasma can support the Langmuir turbulence. This requires that Landau damping, the absorption process corresponding to Cherenkov emission, is ineffective. This effectively limits induced Raman scattering to low 
frequencies unless the plasma is dense and cool:

$$
v<90\left(\frac{n_{e}}{10^{6} \mathrm{~cm}^{-3}}\right)^{1 / 2}\left(\frac{T}{10^{6} \mathrm{~K}}\right)^{-1 / 2} \mathrm{MHz} .
$$

Since the radiation observed from the SHERP was observed at $1.4 \mathrm{GHz}$, it is not surprising that induced Raman scattering does not occur. It thus does not place a stringent limit on the plasma conditions near the emission generation region.

\section{The Intergalactic Medium}

\subsection{Scattering due to turbulence associated with the Intergalactic Medium}

Inhomogeneities in a plasma are capable of temporally smearing an impulsive burst of radio emission. The effect arises due to the refractive index variations inherent to the inhomogeneities. These cause changes in the propagation direction of the incident radiation, steering emission that was otherwise pointed away from the observer back into the line of sight. However, radiation that has been scattered back into the line of sight arrives significantly delayed with respect to radiation that propagates along the most direct path because it must travel a larger distance to the observer. In practice, in the regime of strong scintillation relevant to most observations, the observer receives radiation over a nearly-continuous range of angles (and hence delays), so that the observed signal is a convolution of the intrinsic burst pulse with a smooth smearing kernel.

The $5 \mathrm{~ms}$ SHERP width, associated by Lorimer et al. with temporal smearing due to multipath propagation, probably represents the first detection of sub-pc scale turbulence in the ionized IGM. This is because the scattering time is almost certainly entirely the result of turbulence in the interGalactic medium, and not the interstellar medium of our Galaxy. There is an enormous lever-arm effect which favours the contribution of the ionized IGM over local turbulence. To see this, let us consider some simple results from thin-screen scattering theory, suitably modified from standard scattering theory to account for the fact that spacetime is appreciably curved over the distances under consideration. An extended-medium treatment is desirable for treating these effects properly in the IGM, but the thin-screen treatment suffices for present purposes to illustrate the relevant physics.

The wavefield received by an observer due to a thin screen of material at a redshift $z_{L}$ from the observer is

$$
u(\mathbf{X})=\frac{e^{-i \pi / 2}}{2 \pi r_{\mathrm{F}}^{2}} \int d^{2} \mathbf{x} \exp \left[\frac{i}{2 r_{\mathrm{F}}^{2}}\left(\mathbf{x}-\frac{D_{L S}}{D_{S}} \mathbf{X}\right)^{2}+i \psi(\mathbf{x})\right],
$$

where $\psi$ represents the phase fluctuations as a function of position, $\mathbf{x}$, on the screen, and the Fresnel scale is

$$
r_{\mathrm{F}}^{2}=\frac{D_{L} D_{L S} \lambda}{2 \pi D_{S}\left(1+z_{L}\right)}
$$

where $D_{L}, D_{S}$ and $D_{L S}$ represent the angular diameter distances between the observer and the screen, the observer and the source, and the screen and the source respectively. These equations 
are identical to those that form the basis of gravitational lensing theory, where the correct treatment of curved geometry of spacetime is essential; the only difference is that here the phase delays are driven by small-scale density inhomogeneities in the plasma, rather than distortions in spacetime imposed by the gravitational fields of massive objects.

It is usually (reasonably) assumed that the phase fluctuations are stationary in the wide sense and obey gaussian statistics. Under these assumptions a complete statistical description of the phase fluctuations is furnished by the phase power spectrum or, equivalently, the phase structure function:

$$
D_{\psi}(r)=\left\langle\left[\psi\left(\mathbf{r}+\mathbf{r}^{\prime}\right)-\psi\left(\mathbf{r}^{\prime}\right)\right]^{2}\right\rangle
$$

Observations in a variety of the astrophysical (and terrestrial) plasmas show that the power spectrum of the turbulence follows a power law whose index, $\beta$, closely matches that expected for Kolmogorov turbulence, $\beta=-11 / 3$, and we adopt this value here. It is possible to cast the phase structure function in the simple form

$$
\begin{aligned}
& D_{\psi}(r)=\left(\frac{r}{r_{\text {diff }}}\right)^{-\beta-2}, \quad r>r_{\text {in }}, \\
& D_{\psi}(r) \propto r^{2}, \quad r<r_{\text {in }} .
\end{aligned}
$$

The quantity $r_{\text {in }}$ is the inner scale of the turbulence. The quantity $r_{\text {diff }}$, known as the diffractive length scale, is interpreted as the scale over which the rms phase change on the thin screen is one radian. We have not explicitly specified any dependence on the amplitude of the phase structure function with redshift. However, it is important to remember that there are two strong potential influences on $r_{\text {diff }}$ as a function of the redshift of the scattering material. Since the mean density of the universe scales as $(1+z)^{3}$, one might expect to encounter denser (and thus more strongly scattering) plasmas at higher redshift. Partially counteracting this is the fact that the phase fluctuations in a plasma are proportional to $\lambda$, contributing a factor $\left(1+z_{L}\right)^{-2}$ to the phase structure function. This is, of course, due to the fact that radiation we observe at a wavelength $\lambda$ actually suffered the phase delay at the shorter wavelength $\lambda /\left(1+z_{L}\right)$ when it encountered the scattering screen.

Using equations (4.1) and (4.2) in conjunction with our prescription for the statistical properties of the phase fluctuations, it is possible to show that a point-like object will possess an angular size due to scattering of (cf. Macquart 2004)

$$
\theta_{\text {scat }} \approx \frac{D_{L S}}{k D_{S} r_{\text {diff }}}
$$

and that an impulse will be smeared to a timescale of width

$$
\tau_{\text {scat }} \approx \frac{1}{c k}\left(\frac{r_{\mathrm{F}}}{r_{\text {diff }}}\right)^{2}=\frac{D_{L} D_{L S}}{c D_{S} k^{2} r_{\text {diff }}^{2}\left(1+z_{L}\right)},
$$

where $k=2 \pi / \lambda$ is the wavenumber of the observed radiation.

Equations (4.6) \& (4.7) raise an important point related to the different weighting of the scattering material along the ray path associated with the two effects. The angular size of a scattered object is dominated by material close to the observer, since it is in this region that the ratio $D_{L S} / D_{S}$ 
is close to unity; it is very difficult for material close to the source itself to contribute because $D_{L S} \ll D_{S}$.

On the other hand, material for which the effective distance $D_{\text {eff }}=D_{L} D_{L S} / D_{S}$ reaches a maximum contributes most to the temporal smearing (given equivalent values of $r_{\text {diff }}$ ). Roughly speaking, this occurs where the angular diameter distance to the scattering material is about half the angular diameter distance to the source. The value of $D_{\text {eff }} \sim 1 \mathrm{kpc}$ corresponding to scattering in the Milky Way is extremely small compared to the $\sim 250 \mathrm{Mpc}$ value expected for intergalactic scattering. If local turbulence is to dominate over intergalactic turbulence, the value of $r_{\text {diff }}$ associated with the local plasma must be at $\sim 500$ times smaller than that associated with the IGM.

One can show that this lever arm effect is sufficiently large that one expects the IGM to dominate temporal smearing to the SHERP. Previous simple calculations have suggested the amplitude of the scattering measure (SM) of the ISM relative to the IGM to be $\sim 10^{-4}$ (Goodman 1997). This estimate is based on the relative mean densities of the ISM and the IGM and the path lengths involved, but it does not take into account the possibility that the IGM is highly clumped (which is likely given that most of the baryons should be distributed along the void walls and absent from voids, and are further likely clustered within galaxy groups). Now since $\mathrm{SM} \propto r_{\text {diff }}^{-5 / 3}$ one sees that $r_{\text {diffISM }}$ is expected to exceed $r_{\text {diffIGM }}$ by a ratio of less than $\approx 250$. This is insufficient to overcome the lever arm favouring the IGM contribution over the ISM, and additional clumping in the IGM likely enhances its contribution much further. A further enhancement is provided by the $(1+z)^{3}$ increase in mean IGM electron density with redshift, discussed above.

Having excluded the possibility that our Galaxy's ISM dominates the contribution of IGM, it remains to consider the contribution of the SHERP's host galaxy. Scattering in the host galaxy must overcome the same lever-arm effect that applies to our Galaxy's ISM. To consider the contribution of the host ISM, let us suppose that much of the observed DM is, in fact, due to the host galaxy and not the IGM. The estimated contribution from our Galaxy is $25 \mathrm{pc} \mathrm{cm}^{-3}$, leaving the host galaxy to account for the bulk of the remaining $350 \mathrm{pc} \mathrm{cm}^{-3}$. The case in which the IGM makes a negligible contribution, however, is extreme and would confine the SHERP to much lower distances than originally supposed; Ioka (2003) estimate $\mathrm{DM}_{\mathrm{IGM}}>\mathrm{DM}_{\text {host }}$ for any object at $z>0.03$. Following Lorimer et al. (2007), we therefore take $\mathrm{DM}_{\text {host }}=200 \mathrm{pc} \mathrm{cm}^{-3}$ as the maximum likely host galaxy contribution.

In our own Galaxy one finds (i) $\tau \propto \mathrm{DM}^{2}$ for $\mathrm{DM}<20 \mathrm{pc} \mathrm{cm}^{-3}$ and (ii) $\tau \propto \mathrm{DM}^{4}$ for higher dispersion measures (see, e.g., Boldyrev \& Gwinn 2003). We can use these two scalings to bracket the amount of temporal smearing expected from the host galaxy. Based upon the expected smearing timescale for our Galaxy ${ }^{3}$, the ratio of DMs between our Galaxy and the host implies a smearing time contribution between 3 and $200 \mu$ s for the host galaxy. This fails to account for the observed smearing time by between one and three orders of magnitude, and suggests that the observed temporal smearing is unlikely to be dominated by the host galaxy. However, we caution that the possibility that the host harbours an exceptionally turbulent (but low DM) turbulent region cannot be dismissed. Ultimately, higher S/N observations of other SHERPs will be able to definitively distinguish between IGM and host ISM scattering based on the exact shape of the temporally smeared

\footnotetext{
${ }^{3}$ The NE2001 Galactic scattering model (Cordes \& Lazio 2002; http://rsd-www.nrl.navy.mil/7213/lazio/ne_model/) estimates the smearing time through the line to the SHERP through our own Galaxy to be $0.05 \pm 0.01 \mu \mathrm{s}$.
} 
pulse; the scattered pulse shape can be used to infer basic properties of the distribution of scattering material along the line of sight (see Lee \& Jokipii 1975 as applied to the ISM and Macquart in prep. in the context of the IGM). For the time being we do, however, note that the level of IGM turbulence implied here is consistent with a value suggested by extrapolation of the angular broadening of radio quasars at $z>2$ in the IGM tenatively found in the MASIV survey down to the $z \sim 0.2$ SHERP redshift (Lovell et al. 2007; Lovell et al in prep).

Although the amplitude of the IGM scattering implied here may be larger than might be naïvely expected, it is not excluded by upper limits set by the apparent angular sizes of GRBs at $z \sim 1$. The $5 \mathrm{~ms}$ temporal smearing time, in conjunction with eq. (4.7) and $D_{\text {eff }} \sim 250 \mathrm{Mpc}$, implies $r_{\text {diff }} \sim$ $7 \times 10^{7} \mathrm{~m}$. The thin screen theory discussed here provides only a rough approximation of $r_{\text {diff }}$ and of the implied angular broadening size, $\theta_{\text {scat }} \sim 1 / k r_{\text {diff. }}$. Detailed calculations extended-medium (Macquart, in pr ep.) show that this value implies angular broadening sizes of $20-30 \mu$ as at $1.4 \mathrm{GHz}$ (equivalent to $0.5-0.8 \mu$ as at $8.46 \mathrm{GHz}$ ) which is not inconsistent with apparent $z \sim 1 \mathrm{GRB}$ angular sizes of $\approx 0.4 \mu$ as inferred from diffractive interstellar scintillation at $8.46 \mathrm{GHz}$ (Frail et al. 2000).

The temporal smearing time can be used to roughly estimate the magnetic field strength in the turbulent IGM. Now, if the magnetic field were negligibly small, one would not expect significant fluctuations below the collisional mean free paths of the electrons and protons, which are many orders of magnitude above the scale observed here. However, when a magnetic field is present the plasma density fluctuations are tied to the magnetic field lines on a scale comparable to the proton gyroradius (see Lithwick \& Goldreich 2001) ${ }^{4}$. Above the inner scale of the turbulence, $r_{\text {in }}$, one expects temporal smearing to scale as $\lambda^{4.4}$, and as $\lambda^{4}$ when below it. The observed scaling of the smearing time scale, $\lambda^{4.8 \pm 0.4}$, suggests $r_{\text {diff }}>r_{\text {in }}$. Thus the observation of turbulence on the scales observed here implies a magnetic field $B \gtrsim 4\left(T_{e} / 10^{3} \mathrm{~K}\right)^{1 / 2} \mathrm{nG}$, where $T_{e}$ is the plasma temperature, here reasonably in the range $10^{3} \mathrm{~K}<T_{e}<10^{5} \mathrm{~K}$.

\subsection{Implications for low-frequency extragalactic transient searches}

The temporal smearing timescale is a very strong function of frequency. The amplitude of the phase structure function scales $\propto \lambda^{2}$, so $r_{\text {diff }}$ scales as $\lambda^{-1}$ if it is less than the turbulence inner scale and as $\lambda^{-2 /(-\beta-2)}$ if not. The smearing time thus scales as $\lambda^{4}$ if $r_{\text {diff }}$ is less than $r_{\text {in }}$. For Kolmogorov turbulence the smearing time scales as $\lambda^{4.4}$ if $r_{\text {diff }}$ exceeds the inner turbulence scale.

This sharp frequency scaling spells disaster for proposed searches for extragalactic transients over the frequency range $30 \mathrm{MHz}-120 \mathrm{MHz}$. Taking the most conservative scaling, $\tau \propto \lambda^{4}$, the $5 \mathrm{~ms}$ SHERP smearing time observed at $1.4 \mathrm{GHz}$ implies a smearing time of

$$
\tau_{\text {scat }}=192\left(\frac{v}{100 \mathrm{MHz}}\right)^{-4} \mathrm{~s},
$$

for this object. Given that this object is only $\sim 500 \mathrm{Mpc}$ distant, one can expect far greater smearing times for events detected over cosmological distances, $z \gtrsim 1$. Such a large scattering time renders the emission much harder to detect. Radiation spread over a timescale $\tau / \tau_{\text {intrinsic }} \equiv R$ larger than the intrinsic timescale results in emission a factor of $R$ lower in flux density. Since the $\mathrm{S} / \mathrm{N}$ of the

\footnotetext{
${ }^{4}$ In fact, the actual inner scale of the turbulence is likely set by the larger of the proton gyroradius, and the ion inertial length, but the observation of a significant amount of turbulence on a given scale requires both lengths not to exceed the measured scale.
} 
integrated emission only improves $\propto \tau^{1 / 2}$, the resulting $\mathrm{S} / \mathrm{N}$ of the detection is reduced by a factor $R^{1 / 2}$. Moreover, detection is further impeded by the fact that this $\mathrm{S} / \mathrm{N}$ can only be recovered after the correct DM associated with the signal has been determined. The DM search must be able to detect the object from a signal a factor of $R$ weaker than the original pulse.

Despite the ruinous effects of multipath smearing, not all is lost. Many of the transients one hopes to detect are generated by coherent emission mechanisms, which generally possess steep spectra, and which can compensate for the loss of S/N due to IGM scattering. For example, the SHERP is found to possess a spectral index $S_{v} \propto v^{-4}$ over the detection bandwidth. The prospects of detecting such objects at low frequency are not so unfavourable if their spectra do not turn over above the search frequency.

\section{Conclusions}

We have considered the effect of induced Compton and induced Raman scattering and multipath smearing due to IGM turbulence on the transient object detected by Lorimer et al. (2007).

The observed event duration is almost certainly dominated by temporal smearing due to turbulence in the Intergalactic Medium. While its $5 \mathrm{~ms}$ smearing time is small at the detection frequency of $1.4 \mathrm{GHz}$, the expected smearing time at $100 \mathrm{MHz}$ exceeds 3 minutes. Detections of temporal smearing at frequencies $\sim 1 \mathrm{GHz}$ are an extremely powerful probe of IGM turbulence on sub-pc scales. However, it constitutes an important limitation on the detectability of objects at frequencies below $\sim 300 \mathrm{MHz}$.

\section{References}

[1] Begelman, M.C., Ergun, R.E. \& Rees, M.J., Cyclotron Maser Emission from Blazar Jets?, 2005, Ap.J. 625,51

[2] Boldyrev, S. \& Gwinn. C., Scintillations and Lévy Flights though the Interstellar Medium, 2003, Ap.J., 584, 791

[3] Cordes, J. M. \& Lazio, T. J. W., NE2001. I. A New Model for the Galactic Distribution of Free Electrons and its Fluctuations; 2002, astro-ph/0207156

[4] Lee, L.C. \& Jokipii, J.R., Strong Scintillations in Astrophysics. II. A Theory of Temporal Broadening of Pulses, 1975, Ap.J., 201, 532

[5] Levinson, A. \& Blandford, R.D. Raman scattering in high-radio-brightness astrophysical systems: application to active galactic nuclei 1995, MNRAS, 274, 717

[6] Lorimer, D.R, Bailes, M, McLaughlin, M.A., Narkevic, D.J. \& Crawford, F. A bright millisecond radio burst of extragalactic origin 2007, Science, in press [astro-ph/0709.4301]

[7] Lithwick, Y. \& Goldreich, P., Magnetohydrodynamic turbulence in interstellar plasmas, 2001, ApJ, 562,279

[8] Lovell, J.E.J. et al., MASIV: The Microarcsecond Scintillation-Induced Variability Survey, 2007, ASP Conf. Ser. 365, 279

[9] Luo, Q. \& Melrose, D.B., Curvature maser emission due to field line torsion in pulsar magnetospheres, 1995, MNRAS, 276, 372 
[10] Macquart, J.-P. Scattering of gravitational radiation: Second order moments of the wave amplitude 2004, A\&A, 422, 761 [astro-ph/ 0402661 ]

[11] Macquart, J.-P. On the detectability of prompt coherent gamma-ray burst radio emission, 2007 Ap.J., 658, L1 [astro-ph/0702098]

[12] Moortgat, J. \& Kuijpers, J. Indirect visibility of gravitational waves in magnetohydrodynamic plasmas 2005, in Proc. 22nd Texas Symp. on Relativistic Astrophysics, ed. P. Chen et al. (eConf. C041213:1230; Stanford: Stanford Univ.), 1230 [gr-qc/ 0503074 ]

[13] Sagiv, A. \& Waxman, E. Collective processes in relativistic plasma and their implications for gamma-ray burst afterglows, 2002, ApJ, 574, 861 [astro-ph/0202337]

[14] Usov, V.V. \& Katz, J.J. Low frequency radio pulses from gamma-ray bursts? 2000, A\&A, 364, 655 [astro-ph/0002278]

[15] Wilson, D.B. \& Rees, M.J. Induced Compton scattering in pulsar winds 1978, MNRAS, 185, 297 